\title{
Pneumonia in pregnancy
}

\author{
Delia loana CUDALBA ${ }^{1}$, Brindusa Ana CIMPOCA-RAPTIS ${ }^{1}$, Anca Marina CIOBANU ${ }^{1,2}$, Corina \\ GICA $^{1}$, Radu BOTEZATU ${ }^{1,2}$, Nicolae GICA ${ }^{1,2}$, Gheorghe PELTECU ${ }^{1,2}$, Anca Maria PANAITESCU ${ }^{1,2}$ \\ ${ }^{1}$ Filantropia Clinical Hospital, Bucharest, Romania \\ 2"Carol Davila" University of Medicine and Pharmacy, Bucharest, Romania
}

\begin{abstract}
Pneumonia is a common disease worldwide and the coexistence with pregnancy increase its morbidity and mortality. Physiologic changes in pregnancy can augment susceptibility to pulmonary diseases and change their clinical course. This review article attempts to make an assessment of current information on the risks, maternal and fetal effects, etiology, clinical features and management of pneumonia in pregnancy.

A systematic literature electronic search for journal articles and guidelines regarding pneumonia and respiratory disease in pregnancy was undertaken.

Pathogens, clinical course and treatment of pneumonia in pregnancy is similar to the non-pregnant circumstances, with particular consideration on obstetric and fetal outcomes and drug safety during pregnancy. Prompt diagnosis and treatment reduce potential complications. Vaccination may reduce severity of pneumonia in high-risk pregnant patients.
\end{abstract}

Keywords: pneumonia, pregnancy, respiratory disease, community acquired pneumonia

\section{INTRODUCTION}

Pneumonia is the most common cause of fatal non-obstetric infection in the pregnant patient [1].

Physiologic and immunologic changes specific to pregnancy may enhance the natural history of pneumonia, which may result in greater maternal and fetal risks.

Regardless of pharmacological advances and respiratory care, pneumonia in pregnancy can lead to unfavorable outcomes, both for mother and fetus.

METHODS

A systematic literature electronic search for reviews and journal articles was undertaken using PubMed and the official websites of Obstetrics and Gynecology societies. Search words were "pneumonia", "pregnancy", "respiratory disease". Publications were selected based on accessibility to full paper article, quality evaluation, publication year. The publications used are mentioned in References section.

\section{PHYSIOLOGIC CHANGES IN PREGNANCY THAT PREDISPOSE TO PNEUMONIA}

Pregnant patients have several characteristics specific to pregnancy predisposing them to an increased incidence and risk of complications from pneumonia [2].

\section{Anatomic changes}

Physiological changes of the respiratory tract during pregnancy include the changes of the diafragm, which rises about $4 \mathrm{~cm}$. Even if the thoracic circumference increases, it is not enough in order to reduce the residual lung volumes. Functional residual capacity decreases 
by $20-30 \%$, meanwhile oxygen requirements increase by $20 \%$. These increased oxygen requirements and increased critical closing volumes - the lung volume required by airways to close during expiration, make the pregnant patient more susceptible to severe disease [3]. In pregnancy there is a higher likelihood of aspiration during labor and delivery [4].

\section{Changes in maternal immune status}

Nourishing the fetus and protecting the maternal host can be a challenge for the immune system.

The immunology of pregnancy determines major adaptations. Maternal immune system act in a such way as to prevent the rejection of the genetically and immunologically foreign fetus. Cellular immunity alterations are physiological in pregnancy, especially during the second and the third trimester. The main changes in maternal immune status are decreased lymphocyte proliferative response, reduced lymphokine response to alloantigens, reduced number of helper T cells, diminished cell-mediated proliferative cytotoxicity $[5,6]$. All these changes affect maternal susceptibility to autoimmune and infectious diseases.

\section{Hormonal changes}

The hormonal changes orchestrate the immunological alterations. During pregnancy, several hormones, as progesterone, estradiol, estriol, corticosteroids, prolactin, change their concentrations. They modulate the changes in immune function and disease susceptibility [7].

\section{RISK FACTORS}

Regarding the onset of pneumonia, studies show that the mean gestational age ranges from 24 to 31 weeks. Maternal age and parity don't seem to influence pneumonia occurrence [8]. Conditions associated with a higher rate of infections are anemia, asthma, cystic fibrosis, HIV infection, immunosuppressive diseases and therapy. Furthermore, the use of antepartum corticosteroids, smoking and the use of illicit drug predispose to an increased incidence and mortality [9].

\section{EFFECT OF PNEUMONIA ON MATERNAL AND OBSTETRIC OUTCOME}

Pneumonia can lead to various complications in the non-pregnant patient, as arthritis, meningitis, endocarditis, sepsis, acute respiratory distress syndrome, which can all develop also in the pregnant patient [10]. Pneumonia is not a common cause of death among pregnant women, but puts pregnant women at a higher risk, even after pre-antibiotic era [5].
The most frequent fetal complications reported in studies were premature birth, fetal growth restriction, small for gestational age newborns. Antepartum pneumonia was not associated so far with congenital syndromes, but hypoxemia, fever and tachypnea are likely to lead to an adverse outcome to the fetus [5]. It is acknowledged that maternal and fetal complications have a higher incidence when pneumonia occurs in women with comorbid conditions [10].

\section{ETIOLOGY OF PNEUMONIA IN PREGNANCY}

Studies showed that there is no difference between the etiology of pneumonia occurring in the pregnant patient versus the non-pregnant patient. Common agents were found to be in community-acquired pneumonia, pathogens as Streptococcus pneumoniae, Hemophilus influenzae, atypical bacterial agents such as Legionella, Mycoplasma pneumoniae, Chlamydophila pneumoniae. There are also included viral agents (Influenza A, Varicella and lately SARS-CoV-2, the 2019 novel coronavirus), Pseudomonas aeruginosa, rarely fungi and Coccidioidomycosis, Pneumocystis jiroveci (with HIV infection, especially with a low CD4 cell count) [11]. Often, there is no etiologic agent identified.

The risk of aspiration and further aspiration pneumonia is increased in pregnancy, particularly in the peri-partum period. Patients can aspirate solid or liquid stomach contents or bacteria from the oropharynx. The symptoms include bronchospasm, tachypnea, cough, hypotension, pulmonary edema. In order to prevent such complication, is indicated regional anesthesia over general anesthesia and airway protection [4].

\section{CLINICAL PRESENTATION AND MANAGEMENT}

The clinical findings of pneumonia during pregnancy do not differ substantially from the ones in non-pregnant adults. The typical presentation include fever, chills, dyspnea, pleuritic chest pain, cough, sputum production and hypoxia. Indications for intensive care unit admission are the need for mechanical ventilation, respiratory rate of $>30$ breaths per minute, confusion, platelets $<100,000$ per $\mathrm{mm}^{3}$, white blood cell count $<400$ per $\mathrm{mm}^{3}, \mathrm{~Pa} 2 / \mathrm{FiO} 2$ ratio $<250 \mathrm{mmHg}$. These criteria apply both for the non-pregnant patient and the pregnant one. Moreover, pregnant women have reduced physiological ability to tolerate hypoxia.

Diagnostic testing includes chest radiograph (with an abdominal shield), assessment of gas exchange, blood count, routine blood chemistry. As blood cul- 
tures can give false-positive results, they are indicated only in severe illness. Sputum cultures are recommended if a drug-resistant agent or a pathogen not covered by usual empiric therapy is suspected [4].

\section{TREATMENT}

The treatment of pneumonia in pregnancy is similar to the one administered to a non-pregnant patient, taking into account the well-known antibiotics avoided in pregnancy. First-line therapy is empiric, seeking to cover the common pathogens for community-acquired pneumonia as $S$. pneumoniae, $H$. influenzae for typical bacterial pathogens and $M$. pneumonia, Legionella pneumophila or C. pneumoniae for atypical bacterial pathogens. Therefore, considering the agent's safety and efficacy cephalosporins and macrolides (erythromycin or azithromycin) are the choice for community-acquired pneumonia $[2,4]$.

The fluoroquinolones, commonly used in non-pregnant patients, are avoided because of the risks of arthropathy and malformations proved in animal studies. Other drugs to be avoided are aminoglycosides, secondary to the risk of fetal ototoxicity, tetracyclines because of the association with bony deformities, teeth deformation and staining, maternal fulminant hepatitis, vancomycin due to fetal nephrotoxicity and ototoxicity, chloramphenicol because it can cause circulatory collapse - "gray baby syndrome" (ashen-gray color, flaccidity, cyanosis, abdominal distention) $[2,4]$.

Supportive therapy, as in the non-pregnant patient, comprises hydration, supplemental oxygen, antipyretic therapy. It is a priority maintaining an adequate oxygenation because hypoxemia is less well tolerated in pregnant patients.

\section{PREVENTION OF PNEUMONIA}

Inactivated influenza vaccination can be administered safely during pregnancy, before and during the influenza season, meanwhile undoubtedly live attenuated influenza vaccine is not recommended. The varicella vaccine is a live attenuated vaccine, so it is not recommended for use during pregnancy, but it can be administered at 1-3 months before pregnancy. Regarding pneumococcus infection prevention, there is no pregnancy recommendation published for pneumococcal conjugate and pneumococcal polysaccharide, but if no adverse consequences were reported among newborns whose mothers were inadvertently vaccinated during pregnancy it may be used in women with immuno-compromised states, chronic cardiopulmonary disease or other severe underlying disease $[5,12]$.

\section{DISCUSSION}

The occurrence of pneumonia in pregnancy may be attributed to the mixture of anatomic, immunological, hormonal and independent risk factors. These factors make pregnant patients more susceptible to severe forms of disease.

Some preexisting comorbidities (asthma, HIV, immunosuppressive diseases) could represent risk factors too. Pneumonia could induce many complications to the fetus, such as IUGR, premature delivery, small for gestational age.

Concerning medical treatment, it is important to note that quinolones would not be recommended as first the line treatment because their potential harmful effect on both mother and fetus [13]. They act on synthesis of conjunctive tissue and could affect tendons, joints and great blood vessels. Other potential side effects are on mental health, glycemia (hypoglycemic coma), myasthenia gravis (worsening symptoms), peripheral nerves (irreversible neuropathy) [13]. Fluoroquinolones should be reserved for use in patients with these conditions who have no alternative treatment options [13].

Community-acquired pneumonia is the most frequent form of pneumonia during pregnancy. The consequences are the typical infectious outcomes that could develop also in the non-pregnant. Medical care providers should assess patient's symptoms and conduct and evaluate illness severity, in order to manage safe and effective the proper therapy. Current antibiotic and antiviral therapies can reduce maternal morbidity and mortality and vaccination is part of pneumonia prevention.

\section{CONCLUSIONS}

Pneumonia in pregnancy is associated with increased morbidity and mortality than in the non-pregnant patients. Pregnant women diagnosed with pneumonia have higher risk of premature birth, small for gestational age or low birth weighted newborns and C-section. Early diagnosis and proper management can improve prognosis for both fetus and mother.

Conflict of interest: none declared

Financial support: none declared 


\section{REFERENCES}

1. Kaunitz AM, Hughes JM, Grimes DA, Smith JC, Rochat RW, Kafrissen ME. Causes of maternal mortality in the United States. Obstet Gynecol. 1985 May;65(5):605-12.

2. Mehta N, Chen K, Hardy E, Powrie R. Respiratory disease in pregnancy. Best Pract Res Clin Obstet Gynaecol. 2015 Jul;29(5):598-611.

3. Pulmonary Disorders. In: Cunningham FG, Leveno KJ, Bloom SL, Spong CY, Dashe JS. Williams Obstetrics, 24th edition. New York: McGraw-Hill Education, 2014.

4. Brito V, Niederman MS. Pneumonia complicating pregnancy. Clin Chest Med. 2011 Mar;32(1):121-132.

5. Abu-Raya B, Michalski C, Sadarangani M, Lavoie PM. Maternal Immunological Adaptation During Normal Pregnancy. Front Immunol. 2020 Oct 7;11:575197.
6. Robinson DP, Klein SL. Pregnancy and pregnancy-associated hormones alter immune responses and disease pathogenesis. Horm Behav. 2012 Aug;62(3):263-71.

7. Tang P, Wang J, Song Y. Characteristics and pregnancy outcomes of patients with severe pneumonia complicating pregnancy: a retrospective study of 12 cases and a literature review. BMC Pregnancy Childbirth. 2018 Nov 3;18(1):434.

8. Munn MB, Groome LJ, Atterbury JL, Baker SL, Hoff C. Pneumonia as a complication of pregnancy. J Matern Fetal Med. 1999 Jul-Aug;8(4):151-4.

9. Sheffield JS, Cunningham FG. Communityacquired pneumonia in pregnancy. Obstet Gynecol. 2009 Oct;114(4):915-922.
10. Rodrigues J, Niederman MS. Pneumonia complicating pregnancy. Clin Chest Med. 1992 Dec;13(4):679-91.

11. CDC, August 2016. Guidelines for Vaccinating Pregnant Women.

12. Chen YH, Keller J, Wang IT, Lin CC, Lin HC. Pneumonia and pregnancy outcomes: a nationwide population-based study. Am J Obstet Gynecol. 2012 Oct;207(4):288.e1-7.

13. U.S. Food and Drug Administration - Press Announcements - FDA updates warnings for fluoroquinolone antibiotics on risks of mental health and low blood sugar adverse reactions July 10, 2018. Available at: https:// www.fda.gov/news-events/pressannouncements/fda-updates-warningsfluoroquinolone-antibiotics-risks-mentalhealth-and-low-blood-sugar-adverse. 